\title{
Application of a prediction model with laboratory indexes in the risk stratification of patients with COVID-19
}

\author{
JIRU YE ${ }^{1}$, XIAOQING ZHANG ${ }^{2}$, FENG ZHU $^{2}$ and YAO TANG ${ }^{3}$ \\ ${ }^{1}$ Department of Respiratory and Critical Care Medicine, The Third Affiliated Hospital of Soochow University, \\ Changzhou, Jiangsu 213003; ${ }^{2}$ Department of Respiratory and Critical Care Medicine, Wuxi Fifth People's Hospital, Wuxi, \\ Jiangsu 214000; ${ }^{3}$ Department of Tuberculosis, Huaian No. 4 People's Hospital, Huaian, Jiangsu 223000, P.R. China
}

Received September 10,2020; Accepted December 8, 2020

DOI: $10.3892 /$ etm.2021.9613

\begin{abstract}
In the present study, a prediction model with combined laboratory indexes in risk stratification of patients with COVID-19 was established and tested. The data of 170 patients with COVID-19 who were divided into an asymptomatic-moderate group (141 cases) and severe or above group (29 cases) were retrospectively analyzed. The clinical characteristics and laboratory indexes of the two groups were compared. Multivariate logistic regression analysis was performed to construct the prediction model based on laboratory indexes. A receiver operating characteristic (ROC) curve analysis was used to compare the diagnostic efficacy of different indexes. Decision curve analysis (DCA) was performed to quantify and compare the clinical validity of the prediction models. There were significant differences in blood cell count, high-sensitivity C-reactive protein (hsCRP) and procalcitonin (PCT) levels between the severe or above group and the asymptomatic-moderate group (all $\mathrm{P}<0.05$ ). Among all individual indexes, hsCRP had the highest diagnostic efficacy (area under the curve $=0.870$ ), with a sensitivity and specificity of 0.828 and 0.802 , respectively. The red blood cell count, hsCRP and PCT were used to construct the prediction model. The AUC of the prediction model was higher than that of hsCRP (0.912 vs. 0.870$)$ but the difference was not significant $(\mathrm{P}=0.307)$. DCA suggested that the net benefit of the prediction model was higher than that of hsCRP in most cases and significantly higher than that of PCT, lymphocytes
\end{abstract}

Correspondence to: Professor Feng Zhu, Department of Respiratory and Critical Care Medicine, Wuxi Fifth People's Hospital, 1215 Guangrui Road, Liangxi, Wuxi, Jiangsu 214000, P.R. China

E-mail: 181515688@qq.com

Professor Yao Tang, Department of Tuberculosis, Huaian No. 4 People's Hospital, 128 Yanan East Road, Qingpu, Huaian, Jiangsu 223000, P.R. China

E-mail: 18751264998@163.com

Key words: COVID-19, decision support techniques, C-reactive protein and monocytes. The prediction model with combined laboratory indexes was able to more effectively predict the clinical classification of patients with COVID-19 and may be used as a tool for risk stratification of patients.

\section{Introduction}

Coronavirus disease 2019 (COVID-19) is a novel respiratory and systemic disease caused by severe acute respiratory syndrome coronavirus 2 (SARS-CoV-2). It first appeared in Wuhan (China) and spread rapidly worldwide, bringing great challenges to global public healthcare (1-3). Until July 12020 , $>10$ million people worldwide have been infected with SARS-CoV-2 (4). Most patients with COVID-19 had mild symptoms and their clinical manifestations were mostly fever, fatigue and dry cough, while a small number of patients were critically ill; they developed severe pneumonia and multiple organ failure, which eventually led to death (5-7). Therefore, it is crucial to predict and intervene with the disease as early as possible.

Numerous studies have compared the clinical features and laboratory test results in patients with mild and severe COVID-19 (8-10). Certain prediction models have been developed and studied, including the high-risk population identification model, diagnosis model and prognosis model of COVID-19 infection (11). The predictive factors of most models are the patients' basic characteristics and imaging features (12-14). The present study aimed to establish a simple and effective laboratory index model to predict the clinical classification and risk stratification of patients with COVID-19. This is of great value for the early identification of critically ill patients and these routine laboratory tests are easily available even in resource-poor environments.

\section{Materials and methods}

General data. The present study was a retrospective cross-sectional study. The data of patients with COVID-19 who were hospitalized at the negative pressure ward of Wuxi Fifth People's Hospital (Wuxi, China) from January to April 2020 were analyzed. This study was approved by the Institutional Ethics Committee of Wuxi Fifth People's Hospital (Wuxi, China) for retrospective analysis (approval 
no. 2020-016-1). All data were anonymous. Therefore, informed consent was not required. The inclusion criteria were as follows: i) Novel coronavirus nucleic acid positive as detected by real-time fluorescence reverse transcription PCR; ii) meeting of the diagnostic criteria outlined in the 'Diagnosis and Treatment Protocol for Novel Coronavirus Pneumonia (Trial Version 7)' (15). The exclusion criteria were as follows: i) Pregnant females; ii) patients who did not have a complete medical history, particularly the routine blood test results and detection of infection markers. The general information, complications and the results of routine laboratory tests were collected.

Diagnosis. The clinical classification of patients with COVID-19 was mainly based on symptoms and imaging manifestations. According to the Diagnosis and Treatment Protocol for Novel Coronavirus Pneumonia (trial version 7) published by the General Office of the National Health Commission of China (15), patients with COVID-19 were classified as mild, moderate, severe or critical cases. Patients with mild COVID-19 had mild clinical symptoms and no pneumonia signs on imaging. Moderate cases had fever and respiratory symptoms with imaging findings of pneumonia. Cases meeting any of the following criteria were defined as severe cases: Respiratory distress (respiratory rate, $\geq 30$ breaths/min); oxygen saturation $\leq 93 \%$ at rest; arterial oxygen partial pressure/fraction of inspired oxygen $\leq 300 \mathrm{mmHg}$. Lung imaging indicated that the lesions progressed significantly within 24-48 $\mathrm{h}$ and patients with lung lesions occupying $>50 \%$ of the lung were treated according to management protocols for severe cases. Cases meeting any of the following criteria were defined as critical cases: Respiratory failure and requirement of mechanical ventilation; shock; combination with failure of other organs that required care at the intensive care unit.

Laboratory tests. The following parameters were analyzed: White blood cells (reference range, 3.5-9.5 $\times 10^{9} / 1$ ), the percentage of neutrophils (Neur; reference range, 40.00-75.00\%), neutrophil count (Neuc; reference range, 1.8-6.3 $\times 10^{9} / 1$ ), percentage of lymphocytes (Lymr; reference range, 20.00-50.00\%), lymphocyte count (Lymc; reference range, 1.10-3.20x10 $/ 1$ ), percentage of monocytes (Monr; reference range, 3.00-8.00\%), monocyte count (Monc; reference range, $\left.0.10-0.60 \times 10^{9} / 1\right)$, red blood cell count (RBC; reference range for females and males, 3.80-5.10 and 4.30-5.80×10 $12 / 1$, respectively), hemoglobin (HGB; reference range for females and males, 115-150 and 130-17 $5 \mathrm{~g} / 1$, respectively), hematocrit (HCT; reference range for females and males, 35.0-45.0 and 40.0-50.0\%, respectively), platelet count (reference range, 125-350x 10 $/ 1$ ), red blood cell distribution width (reference range, 11.50-14.90\%), plateletcrit (PTC; reference range, 0.108-0.272 1/1), mean platelet volume (reference range, 6.00-11.50 fl), platelet distribution width (reference range, 15.50-18.10 fl), high-sensitivity C-reactive protein (hsCRP; reference range, $0-10 \mathrm{mg} / \mathrm{l}$ ), procalcitonin (PCT; reference range, $0-0.05 \mathrm{ng} / \mathrm{ml}$ ). A routine blood test was performed using a Sysmex XN9000 blood analyzer (Sysmex Corp.). The hsCRP was detected using a specific protein analyzer HP-083/4 (Hipro Biotechnology). PCT was determined using the Autobio A2000PLUS automatic chemiluminescence instrument (Sym-Biotechnology).
Statistical analysis. Continuous variables are expressed as the mean \pm standard deviation, while categorical variables were expressed as n (\%). An unpaired Student's t-test or Mann-Whitney U-test as nonparametric tests were used to compare continuous variables. Pearson's chi-square test and Fisher's exact test were used to analyze categorical variables.

Multivariate logistic regression analysis was used to establish the prediction model and the best model parameters were selected according to the minimum Akaike's information criterion. The nomogram and a calibration curve of the model were generated. Receiver operating characteristic (ROC) curves were created for each model. The area under the ROC curve (AUC) of different models was compared using the DeLong method (16). The bootstrap resampling method (times=500) recommended by the TRIPOD statement (14) was used to verify the model internally and calculate the 95\% confidence interval (CI) of the AUC. Decision curve analysis (DCA) was used to quantify and compare the clinical effectiveness of the model. This method was based on the threshold probability to express the relative hazards of false-positives and false-negatives. The net benefit was obtained by subtracting the proportion of false-positive results from the proportion of true-positive results and weighing the relative hazards of false-positive and false-negative results. The following formula was used to calculate the net benefit of making decisions based on the model: Net benefit=True positives/n-[Pt/(1-Pt)] x [False positives/n], where $n$ is the total number of patients in the study and $\mathrm{Pt}$ is the given threshold probability.

Finally, stratification analysis was performed to evaluate the possible effects of different subgroups on the prediction efficiency of the combined indexes model. All analyses were performed using $\mathrm{R}$ software, version 3.4.3 (http://www.R-project.org). $\mathrm{P}<0.05$ was considered to indicate statistical significance.

\section{Results}

Patients. A total of 170 patients with COVID-19 were enrolled in the present study, including 94 males and 76 females with an average age of $44.7 \pm 17.8$ years (range, 5-91 years). According to the symptoms and imaging findings on admission, there were 13 asymptomatic cases, 37 mild cases, 91 moderate cases, 27 severe cases and 2 critical cases (15 patients were eventually classified as severe cases because they converted from moderate to severe at 2 days after admission). According to the treatment's clinical significance, the patients were divided into two groups: The asymptomatic-moderate group (141 cases) and the severe or above group (29 cases). A comparison of the general information between the two groups is provided in Table I. It was indicated that in the severe or above group, the age was higher $(\mathrm{P}<0.001)$ and the proportion of cases combined with hypertension, diabetes mellitus and cerebrovascular disease was higher $(\mathrm{P}<0.05)$. The proportion of patients with a tumor history was also higher in this group but the difference was not statistically significant $(\mathrm{P}=0.076)$.

Laboratory data. A comparison of the laboratory indexes between the two groups is provided in Table II. After adjusting for age, gender, hypertension, diabetes mellitus, 
Table I. Comparison of the general data between the asymptomatic-moderate group and the severe or above group.

\begin{tabular}{|c|c|c|c|}
\hline Clinical classification & $\begin{array}{l}\text { Asymptomatic-moderate group } \\
\qquad(\mathrm{n}=141)\end{array}$ & $\begin{array}{l}\text { Severe or above group } \\
\qquad(\mathrm{n}=29)\end{array}$ & P-value \\
\hline Age (years) & $41.6 \pm 16.9$ & $59.6 \pm 14.8$ & $<0.001$ \\
\hline Age group (years) & & & $<0.001$ \\
\hline$<60$ & $123(87.2)$ & 17 (58.6) & \\
\hline$\geq 60$ & $18(12.8)$ & $12(41.4)$ & \\
\hline Sex & & & 0.224 \\
\hline Female & $66(46.8)$ & $10(34.5)$ & \\
\hline Male & $75(53.2)$ & $19(65.5)$ & \\
\hline Hypertension & $31(22.0)$ & $12(41.4)$ & 0.029 \\
\hline Diabetes mellitus & $15(10.6)$ & $10(34.5)$ & $<0.001$ \\
\hline Coronary heart disease & $3(2.1)$ & $2(6.9)$ & 0.166 \\
\hline Cerebrovascular disease & $0(0.0)$ & $2(6.9)$ & 0.002 \\
\hline History of tumor & $1(0.7)$ & $2(6.9)$ & 0.076 \\
\hline $\mathrm{HBV}$ & $1(0.7)$ & $0(0.0)$ & 0.649 \\
\hline Chronic renal disease & $1(0.7)$ & $1(3.4)$ & 0.215 \\
\hline Chronic liver disease/cirrhosis & $3(2.1)$ & $0(0.0)$ & 0.428 \\
\hline Alcoholism & $4(4.2)$ & $1(3.4)$ & 0.855 \\
\hline Smoking & $5(5.3)$ & $2(6.9)$ & 0.739 \\
\hline
\end{tabular}

Values are expressed as the mean \pm standard deviation or $\mathrm{n}(\%)$. HBV, Hepatitis B virus.

Table II. Comparison of laboratory indexes between the asymptomatic-moderate group and the severe or above group.

\begin{tabular}{|c|c|c|c|c|c|}
\hline Laboratory index & Reference values & $\begin{array}{l}\text { Asymptomatic-moderate } \\
\text { group }(n=141)\end{array}$ & $\begin{array}{l}\text { Severe or above } \\
\quad(n=29)\end{array}$ & OR $(95 \% \mathrm{CI})$ & P-value \\
\hline $\mathrm{WBC}\left(\mathrm{x} 10^{9} / \mathrm{l}\right)$ & $3.5-9.5$ & $5.0 \pm 1.5$ & $5.5 \pm 2.7$ & $1.190(0.937,1.513)$ & 0.154 \\
\hline Neur $(\%)$ & $40.0-75.0$ & $58.8 \pm 11.9$ & $70.5 \pm 16.1$ & $1.072(1.026,1.120)$ & 0.002 \\
\hline Lymr (\%) & $20.0-50.0$ & $30.4 \pm 11.1$ & $21.1 \pm 12.6$ & $0.935(0.888,0.985)$ & 0.012 \\
\hline Monr (\%) & $3.0-8.0$ & $9.6 \pm 3.2$ & $7.5 \pm 3.7$ & $0.762(0.633,0.916)$ & 0.004 \\
\hline Neuc $\left(\times 10^{9} / 1\right)$ & $1.8-6.3$ & $3.0 \pm 1.3$ & $4.1 \pm 2.8$ & $1.375(1.042,1.815)$ & 0.025 \\
\hline Lymc $\left(x 10^{9} / 1\right)$ & $1.1-3.2$ & $1.5 \pm 0.7$ & $1.0 \pm 0.5$ & $0.300(0.099,0.911)$ & 0.034 \\
\hline Monc (x109/1) & $0.1-0.6$ & $0.5 \pm 0.2$ & $0.4 \pm 0.2$ & $0.058(0.003,1.018)$ & 0.051 \\
\hline $\mathrm{RBC}\left(\mathrm{x} 10^{12} / \mathrm{l}\right)$ & $\begin{array}{l}\text { Females, 3.8-5.1; } \\
\text { males, 4.3-5.8 }\end{array}$ & $4.7 \pm 0.7$ & $4.3 \pm 0.6$ & $0.413(0.148,1.151)$ & 0.091 \\
\hline $\operatorname{HGB}(\mathrm{g} / \mathrm{l})$ & $\begin{array}{l}\text { Females, 115-150; } \\
\text { males, 130-175 }\end{array}$ & $139.6 \pm 17.9$ & $130.1 \pm 21.2$ & $0.973(0.946,1.002)$ & 0.066 \\
\hline $\operatorname{HCT}(\%)$ & $\begin{array}{l}\text { Females, 35.0-45.0; } \\
\text { males, 40.0-50.0 }\end{array}$ & $41.2 \pm 4.7$ & $37.7 \pm 5.5$ & $0.868(0.775,0.972)$ & 0.014 \\
\hline $\operatorname{PLT}\left(\times 10^{9} / 1\right)$ & $125.0-350.0$ & $184.3 \pm 58.5$ & $173.9 \pm 56.5$ & $1.006(0.997,1.015)$ & 0.173 \\
\hline RDW (\%) & $11.5-14.9$ & $13.1 \pm 1.9$ & $13.6 \pm 2.8$ & $1.191(0.871,1.629)$ & 0.274 \\
\hline MPV (fl) & $6.0-11.5$ & $11.0 \pm 1.2$ & $10.9 \pm 1.2$ & $0.734(0.467,1.155)$ & 0.182 \\
\hline PDW (fl) & $15.5-18.1$ & $14.8 \pm 3.2$ & $15.3 \pm 2.1$ & $0.980(0.806,1.192)$ & 0.840 \\
\hline PTC (1/l) & $0.108-0.272$ & $0.2 \pm 0.1$ & $0.2 \pm 0.1$ & $0.904(0.000,10,957.021)$ & 0.983 \\
\hline hsCRP (mg/l) & $0.0-10.0$ & $13.6 \pm 21.0$ & $78.3 \pm 63.8$ & $1.041(1.020,1.062)$ & $<0.001$ \\
\hline PCT (ng/ml) & $0.00-0.05$ & $0.15 \pm 0.18$ & $0.46 \pm 0.64$ & $13.774(1.399,135.619)$ & 0.025 \\
\hline
\end{tabular}

Adjusted variables: Age, sex, hypertension, diabetes mellitus, cerebrovascular diseases, history of the tumor. OR, odds ratio; WBC, white blood cells; Neur, percentage of neutrophils; Lymr, percentage of lymphocytes; Monr, percentage of monocytes; Neuc, neutrophil count; Lymc, lymphocyte count; Monc, monocyte count; RBC, red blood cell count; HGB, hemoglobin; HCT, hematocrit, PLT, platelet count; RDW, red blood cell distribution width; MPV, mean platelet volume; PDW, platelet distribution width; PTC, plateletcrit; hsCRP, high-sensitivity C-reactive protein; PCT, procalcitonin. 


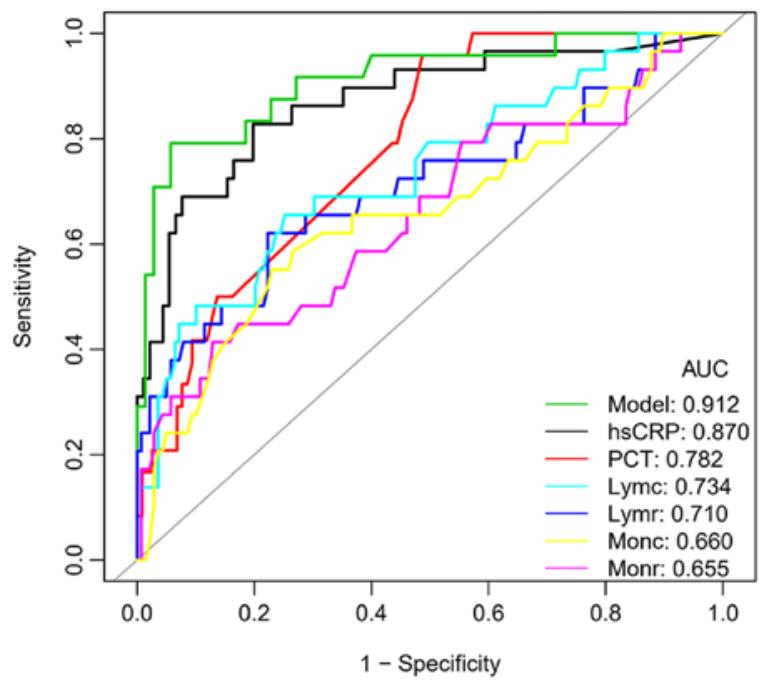

Figure 1. ROC curves of different indexes used for the clinical classification of patients with COVID-19. hsCRP, high-sensitivity C-reactive protein; PCT, procalcitonin; Lymc, lymphocyte count; Lymr, percentage of lymphocytes; Monc, monocyte count; Monr, percentage of monocytes; AUC, area under the ROC curve; ROC, receiver operating characteristic.

cerebrovascular disease and tumor history, it was revealed that the Neur, Neuc, hsCRP and PCT in the severe or above group were significantly higher than those in the asymptomatic-moderate group (all $\mathrm{P}<0.05$ ), while the Lymr, Monr, Lymc and HCT in the severe or above group were significantly lower than those in the asymptomatic-moderate group (all $\mathrm{P}<0.05$ ).

Predictive value of individual laboratory indexes. The Diagnosis and Treatment Protocol for Novel Coronavirus Pneumonia (trial version 7) (15) pointed out that the peripheral blood lymphocytes of patients with severe COVID-19 were progressively reduced. Therefore, ROC curves were generated for the Lymr, Lymc, Monr and Monc in the two groups. The cut-off value, AUC and 95\% CI of the above four indexes were 22.10, 0.710 (0.591-0.828); 1.02, 0.734 (0.626-0.842); 6.45, $0.655(0.535-0.776)$ and $0.34,0.660(0.540-0.780)$, respectively, and the AUC of Lymc was the largest (Fig. 1, Table III).

Construction of prediction model using combined laboratory indexes. Multivariate logistic regression analysis was performed using whether the patient was diagnosed as a severe case or above as the dependent variable and laboratory indexes, including the Neur, Lymr, Monr, Neuc, Lymc, Monc, RBC, HGB, HCT, hsCRP and PCT, as independent variables (Table II; P-value threshold was extended to 0.10) to establish the risk prediction model. It was indicated that RBC, hsCRP and PCT were independent risk factors for severe disease. The prediction model formula established was as follows: $\operatorname{Logit}(\mathrm{P})=6.88787-2.36962 \times \mathrm{RBC}\left(\mathrm{x} 10^{12} / 1\right)+0.04830 \times$ hsCRP $(\mathrm{mg} / \mathrm{l})+2.62161 \times \mathrm{PCT}(\mathrm{ng} / \mathrm{ml})$, where the P-value referred to the probability that the patient was diagnosed as a severe case or above.

Next, the nomogram, calibration curve and ROC curve of the prediction model were generated (Fig. 2A-C). It was revealed that the predicted value and the observed value were in agreement with each other.
When comparing the ROC curves of different laboratory indexes and the model with combined indexes for the prediction of clinical classifications of patients with COVID-19 (Table III), it was revealed that the AUC value of the prediction model was higher than that of hsCRP (0.912 vs. 0.870 , respectively), but the difference was not statistically significant $(\mathrm{P}=0.307)$. However, the AUC of the model was significantly higher than that of PCT, lymphocytes and monocytes (all $\mathrm{P}<0.001$ ). The decision curves of different indexes and the prediction model were further generated (Fig. 3). In most cases, the model's net benefit was higher than that of hsCRP and was significantly higher than that of PCT, lymphocytes and monocytes.

Comparison of the laboratory index model in different subgroups. The prediction model indicated a similar trend in different age, gender, hypertension and diabetes subgroups and was a risk factor among different subgroups (all $\mathrm{P}<0.05$ ), and the interaction was not significant (all $\mathrm{P}>0.05$; Table IV). As the number of cases in the coronary heart disease, cerebrovascular disease, tumor, Hepatitis B virus (HBV), chronic kidney disease (CKD) and chronic liver disease/liver cirrhosis subgroups was $<20$ cases, they were not included in the comparison.

\section{Discussion}

The present study mainly discussed the differences between the clinical characteristics, blood routine results and routine inflammatory indexes between mild and severe cases and a prediction model based on laboratory indexes was constructed to predict the clinical classification of patients with COVID-19. These laboratory indexes are simple and easy to obtain and are quantitative indexes so that clinicians are able to evaluate the patient's condition in a timely and effective manner.

The present study indicated that older people and patients with underlying diseases, such as diabetes and hypertension, had a higher risk of severe COVID-19, which was consistent with most studies (17-19). Su et al (20) also pointed out that $19 \%$ of the hospitalized patients with COVID-19 had diabetes mellitus and diabetes mellitus is a risk factor of poor prognosis for the disease.

The present results also suggested that neutrophils increased significantly in patients with severe COVID-19, while lymphocytes, monocytes and hematocrit decreased significantly, consistent with most previous studies (21-24). A meta-analysis by Soraya and Ulhaq (25) suggested that progressive lymphopenia is an important marker for the severity of COVID-19, indicating that viral infection damages the patients' immune system. The present results indicated that the lymphocyte count had the highest diagnostic efficacy for severe COVID-19 (AUC=0.734), which was slightly lower than the result of Soraya and Ulhaq (25) (AUC $=0.870)$, but it was not an independent risk factor for severe disease. $\mathrm{RBC}$ are critical for oxygen transport and off-loading and Thomas et al (26) reported that angiotensin and angiotensin converting enzyme 2 receptor interacting proteins were identified on the RBC surface via proteomics. This indicated that $\mathrm{RBC}$, which theoretically do not support viral replication, may be invaded by SARS-CoV-2, thereby leading to the alteration of the RBC form and their destruction, resulting in hypoxemia in patients and multiple organ failure in severe cases. The results of the present study 
Table III. Comparison of the diagnostic efficacy of different laboratory indexes and the prediction model with combined indexes.

\begin{tabular}{lrcccccc}
\hline Index & Cut-off & AUC $(95 \%$ CI $)$ & Specificity & Sensitivity & Accuracy & Positive-LR & Negative-LR \\
\hline Lymr $(\%)$ & 22.10 & $0.710(0.591-0.828)$ & 0.777 & 0.621 & 0.750 & 2.783 & 0.488 \\
Lymc (x 10 $/ 1)$ & 1.02 & $0.734(0.626-0.842)$ & 0.748 & 0.655 & 0.732 & 2.602 & 0.461 \\
Monr (\%) & 6.45 & $0.655(0.535-0.776)$ & 0.871 & 0.414 & 0.792 & 3.195 & 0.673 \\
Monc (x 10/l) & 0.34 & $0.660(0.540-0.780)$ & 0.770 & 0.552 & 0.732 & 2.397 & 0.582 \\
PCT (ng/ml) & 0.15 & $0.782(0.696-0.868)$ & 0.513 & 0.958 & 0.589 & 1.967 & 0.081 \\
hsCRP (mg/l) & 19.32 & $0.870(0.788-0.952)$ & 0.802 & 0.828 & 0.808 & 4.184 & 0.215 \\
Model & -0.85 & $0.912(0.839-0.985)$ & 0.943 & 0.792 & 0.904 & 13.854 & 0.221 \\
\hline
\end{tabular}

Lymr, percentage of lymphocytes; Lymc, lymphocyte count; Monr, percentage of monocytes; Monc, monocyte count; PCT, procalcitonin; hsCRP, high-sensitivity C-reactive protein; AUC, area under the curve; LR, likelihood ratio.
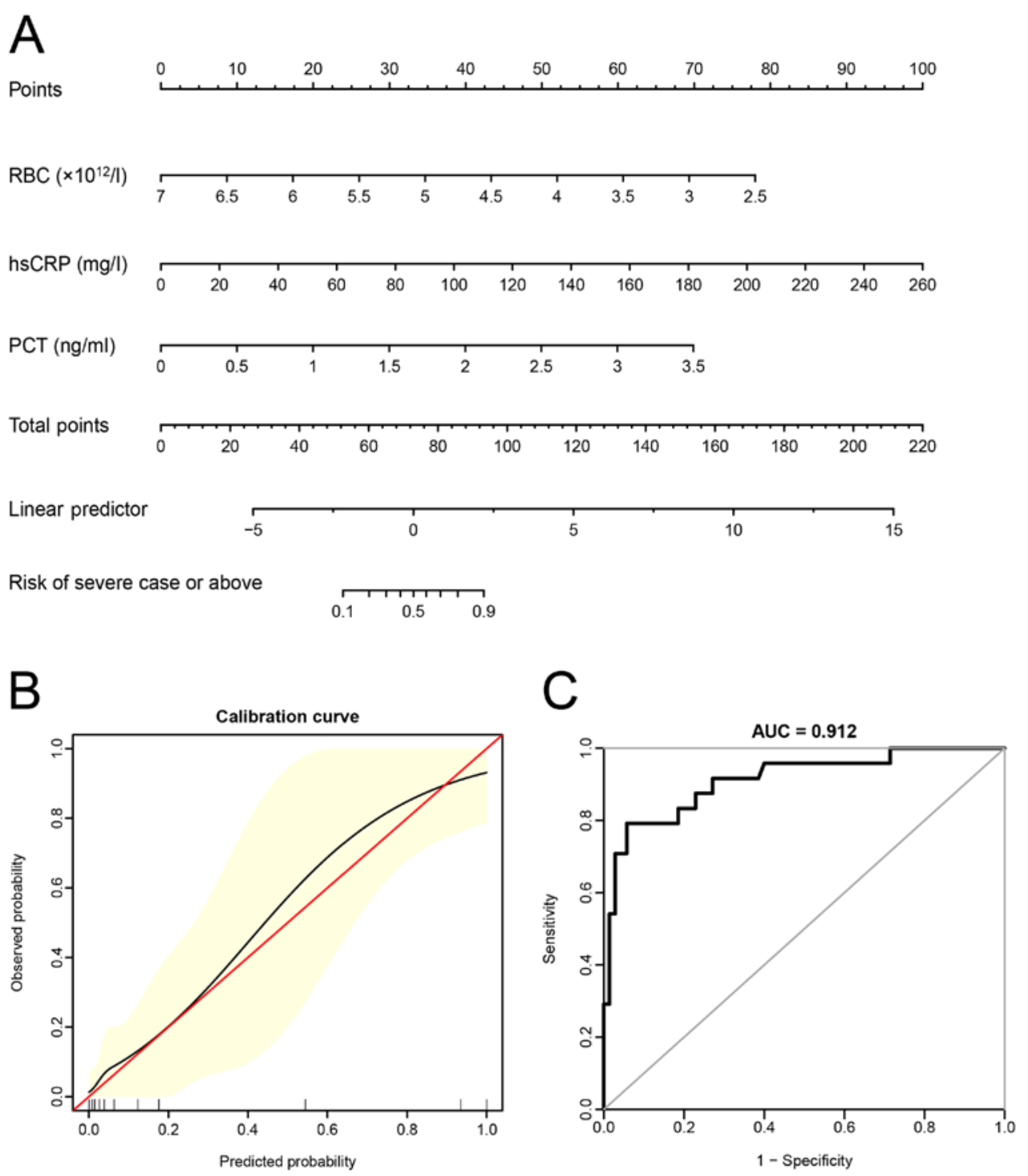

Figure 2. (A) Nomogram, (B) calibration curve and (C) ROC curve of the laboratory index model. RBC, red blood cell count; hsCRP, high-sensitivity C-reactive protein; PCT, procalcitonin; AUC, area under the ROC curve; ROC, receiver operating characteristic.

indicated that this low-cost and routine hematological index may help diagnose and monitor the progress of COVID-19 and should be continuously evaluated during SARS-CoV-2 infection. The present study also suggested that hsCRP and PCT are risk factors for the progression of COVID-19 to severe disease. It has been reported that hsCRP has a significant 
Table IV. Comparison of the efficacy of the prediction model in different subgroups by age, sex, hypertension and diabetes.

\begin{tabular}{lrcc}
\hline Item & $\mathrm{N}$ & OR $(95 \% \mathrm{CI})$ & P-value \\
\hline $\begin{array}{l}\text { Age group (years) } \\
<60\end{array}$ & & & 0.001 \\
$\geq 60$ & 140 & $2.070(1.363-3.142)$ & 0.021 \\
Sex & 30 & $2.959(1.180-7.423)$ & 0.452 \\
Female & & & 0.009 \\
Male & 76 & $2.007(1.195-3.370)$ & $<0.001$ \\
Hypertension & 94 & $2.579(1.527-4.354)$ & $<.507$ \\
No & & & 0.001 \\
Yes & 127 & $2.060(1.387-3.062)$ & 0.394 \\
Diabetes mellitus & 43 & $2.974(1.339-6.602)$ & $<0.001$ \\
No & & & 0.049 \\
Yes & 145 & $2.636(1.628-4.269)$ & 0.282 \\
\hline
\end{tabular}

OR, odds ratio.

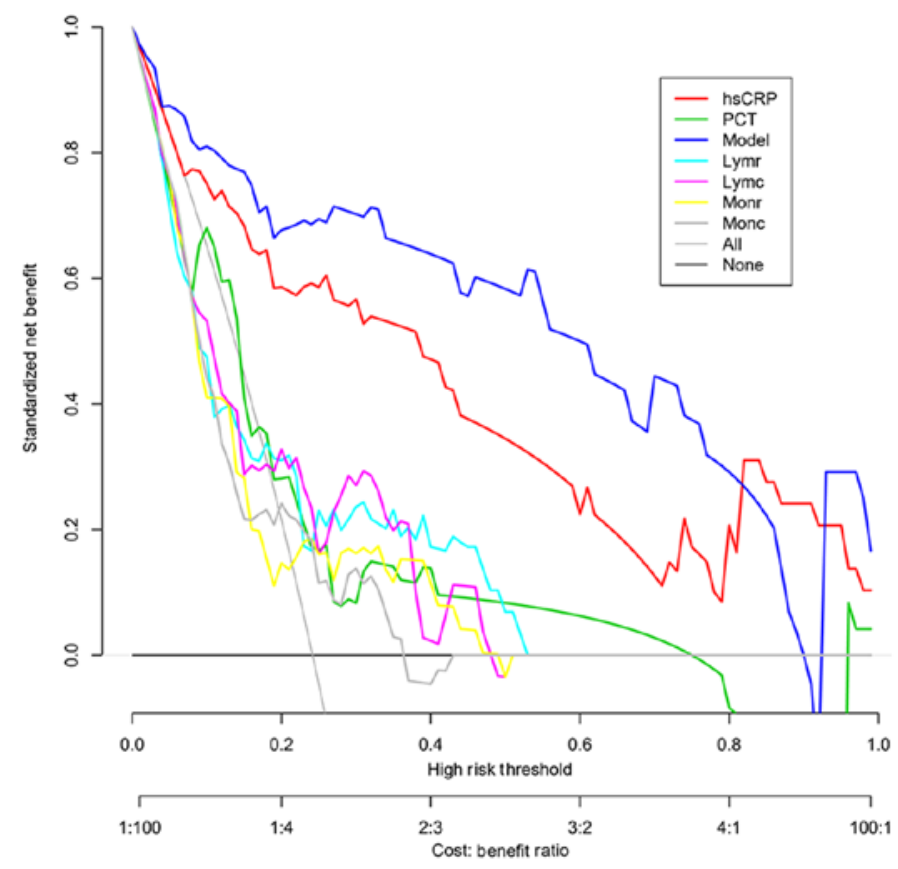

Figure 3. Decision curves of different laboratory indexes and the prediction model. hsCRP, high-sensitivity C-reactive protein; PCT, procalcitonin; Lymr, percentage of lymphocytes; Lymc, lymphocyte count; Monr, percentage of monocytes; Monc, monocyte count.

role in predicting the progression of COVID-19 (27). PCT is a useful marker for evaluating the severity and prognosis of sepsis (28), which helps guide antibiotic treatment. Patients with severe COVID-19 have poor immune function, which increases the risk of secondary infection. Elevated levels of hsCRP and PCT in patients with severe COVID-19 may indicate mixed bacterial infection (29-31). However, in the literature, no significant change in the PCT levels in patients with severe COVID-19 was reported (25). Therefore, future studies with an increased sample size should be performed to investigate this issue further.

Studies have indicated that certain routine laboratory tests are associated with the severity of COVID-19 (32,33).
Although certain individual laboratory indexes may help identify severe cases, the AUC, specificity and sensitivity are generally low $(5,17)$. Therefore, it is urgent to construct a multi-factor prediction model to evaluate patients with COVID-19 effectively. At present, the prediction models that draw attention include a risk model for identifying a high-risk population with SARS-CoV-2 infection within the general population, a diagnosis model for the identification of suspected COVID-19 cases and a prognosis model for predicting the course of infection in patients with COVID-19 (11). Most of the models are based on clinical and imaging features (12-14). To identify patients with severe COVID-19 early, a prediction model based on three quantitative laboratory indexes was 
established: RBC, hsCRP and PCT. Although there was no significant difference in the AUC between this prediction model and hsCRP, the model's specificity was markedly higher (0.943), which was conducive to diagnosing patients with severe COVID-19; it was not affected by age, gender, hypertension or diabetes. In addition, DCA suggested that the clinical benefit of the prediction model with combined laboratory indexes was better than that of hsCRP in most cases. In the present study, certain patients changed from moderate to severe cases, which further highlights the importance of finding good prediction models. Given the common clinical manifestations of viral pneumonia in patients with COVID-19, the progress of general diseases is also short-term (most of the time will appear within a week, even in a week or less), and for such patients, the prediction model may be used to re-evaluate the corresponding indicators in the short term, which can more effectively predict the clinical classification of COVID-19 patients.

The present study had certain limitations. First, it was a retrospective, single-center study; therefore, there may have been a patient selection bias. Furthermore, due to insufficient sample size, the number of cases in subgroups of coronary heart disease, cerebrovascular disease, tumor, HBV, CKD and chronic liver disease/liver cirrhosis is $<20$; thus, stratified validation cannot be performed. In addition, the prediction model was only subjected to internal verification and the feasibility of the model is required to be further verified using external data. Finally, the applicability of the model in 15 patients who had changed from moderate to severe within 2 days after admission was not independently assessed and the period of re-evaluation of laboratory indicators was not taken into account.

In conclusion, in the present study, a prediction model with the following laboratory indexes was constructed: RBC, hsCRP and PCT. This model was able to more effectively predict patients' clinical classification with COVID-19 and may be used as a tool for risk stratification, which is conducive to allocating medical resources and improving the treatment outcome in patients with COVID-19.

\section{Acknowledgements}

Not applicable.

\section{Funding}

The present study was supported by the Young Talent Development Plan of Changzhou Health Commission (grant no. CZQM2020039) and the Youth Foundation Program of Nanjing Medical University (grant no. 2016NJMU170).

\section{Availability of data and materials}

The datasets used and/or analyzed during the current study are available from the corresponding author on reasonable request.

\section{Authors' contributions}

JY drafted the manuscript. JY, FZ and YT made substantial contributions to the conception and design of the study. XZ and YT participated in the acquisition, analysis or interpretation of the data. XZ and YT confirm the authenticity of the raw data. All authors read and approved the final manuscript.

\section{Ethics approval and consent to participate}

This study was approved by the Institutional Ethics Committee of Wuxi Fifth People's Hospital (Wuxi, China; approval no. 2020-016-1). As it was a retrospective analysis and all data were anonymous, no informed consent was required.

\section{Patient consent for publication}

Not applicable.

\section{Competing interests}

The authors declare that they have no competing interests.

\section{References}

1. Fan Z, Chen L, Li J, Cheng X, Yang J, Tian C, Zhang Y, Huang S Liu Z and Cheng J: Clinical features of COVID-19-related liver functional abnormality. Clin Gastroenterol Hepatol 18: 1561-1566, 2020.

2. Wu J, Song S, Cao HC and Li LJ: Liver diseases in COVID-19: Etiology, treatment and prognosis. World J Gastroenterol 26: 2286-2293, 2020.

3. Martins-Filho PR, Tavares CSS and Santos VS: Factors associated with mortality in patients with COVID-19. A quantitative evidence synthesis of clinical and laboratory data. Eur J Intern Med 76: 97-99, 2020.

4. Wiersinga WJ, Rhodes A, Cheng AC, Peacock SJ and Prescott HC: Pathophysiology, transmission, diagnosis, and treatment of coronavirus disease 2019 (COVID-19): A review. JAMA 324: 782-793, 2020.

5. Liu Q, Song NC, Zheng ZK, Li JS and Li SK: Laboratory findings and a combined multifactorial approach to predict death in critically ill patients with COVID-19: A retrospective study. Epidemiol Infect 148: e129, 2020.

6. Wang F, Hou H, Luo Y, Tang G, Wu S, Huang M, Liu W, Zhu Y, Lin Q, Mao L, et al: The laboratory tests and host immunity of COVID-19 patients with different severity of illness. JCI Insight 5: e137799, 2020.

7. Vultaggio A, Vivarelli E, Virgili G, Lucenteforte E, Bartoloni A, Nozzoli C, Morettini A, Berni A, Malandrino D, Rossi $\mathrm{O}$, et al: Prompt predicting of early clinical deterioration of moderate-to-severe COVID-19 patients: Usefulness of a combined score using IL-6 in a preliminary study. J Allergy Clin Immunol Pract 8: 2575-2581.e2, 2020.

8. Chen G, Wu D, Guo W, Cao Y, Huang D, Wang H, Wang T, Zhang X, Chen $\mathrm{H}$, Yu H, et al: Clinical and immunological features of severe and moderate coronavirus disease 2019. J Clin Invest 130: 2620-2629, 2020.

9. Rodriguez-Morales AJ, Cardona-Ospina JA, Gutiérrez-Ocampo E, Villamizar-Peña R, Holguin-Rivera Y, Escalera-Antezana JP, Alvarado-Arnez LE, Bonilla-Aldana DK, Franco-Paredes C, Henao-Martinez AF, et al: Clinical, laboratory and imaging features of COVID-19: A systematic review and meta-analysis. Travel Med Infect Dis 34: 101623, 2020.

10. Zhang B, Zhang J, Chen H, Chen L, Chen Q, Li M, Chen Z, You J, Yang K and Zhang S: Novel coronavirus disease 2019 (COVID-19): Relationship between chest CT scores and laboratory parameters. Eur J Nucl Med Mol Imaging 47: 2083-2089, 2020.

11. Wynants L, Van Calster B, Collins GS, Riley RD, Heinze G, Schuit E, Bonten MMJ, Damen JAA, Debray TPA, De Vos M, et al: Prediction models for diagnosis and prognosis of covid-19 infection: Systematic review and critical appraisal. BMJ 369: $\mathrm{m} 1328,2020$.

12. Lorente-Ros A, Monteagudo Ruiz JM, Rincón LM, Ortega Pérez R, Rivas S, Martínez-Moya R, Sanromán MA, Manzano L, Alonso GL, Ibáñez B and Zamorano JL: Myocardial injury determination improves risk stratification and predicts mortality in COVID-19 patients. Cardiol J 27: 489-496, 2020. 
13. Qin L, Yang Y, Cao Q, Cheng Z, Wang X, Sun Q, Yan F, Qu J and Yang W: A predictive model and scoring system combining clinical and CT characteristics for the diagnosis of COVID-19. Eur Radiol 30: 6797-6807, 2020.

14. Chen X, Tang Y, Mo Y, Li S, Lin D, Yang Z, Yang Z, Sun H, Qiu J, Liao Y, et al: A diagnostic model for coronavirus disease 2019 (COVID-19) based on radiological semantic and clinical features: A multi-center study. Eur Radiol 30: 4893-4902, 2020.

15. Released by National Health Commission \& National Administration of Traditional Chinese Medicine on March 3, 2020: Diagnosis and treatment protocol for novel coronavirus pneumonia (Trial Version 7). Chin Med J (Engl) 133: 1087-1095, 2020.

16. DeLong ER, DeLong DM and Clarke-Pearson DL: Comparing the areas under two or more correlated receiver operating characteristic curves: A nonparametric approach. Biometrics 44: 837-845, 1988.

17. Liu YP, Li GM, He J, Liu Y, Li M, Zhang R, Li YL, Wu YZ and Diao B: Combined use of the neutrophil-to-lymphocyte ratio and CRP to predict 7-day disease severity in 84 hospitalized patients with COVID-19 pneumonia: A retrospective cohort study. Ann Transl Med 8: 635, 2020.

18. Su TH and Kao JH: The clinical manifestations and management of COVID-19-related liver injury. J Formos Med Assoc 119: 1016-1018, 2020

19. Hu L, Chen S, Fu Y, Gao Z, Long H, Ren HW, Zuo Y, Wang J, $\mathrm{Li} \mathrm{H}, \mathrm{Xu} \mathrm{QB}$, et al: Risk factors associated with clinical outcomes in 323 coronavirus disease 2019 (COVID-19) hospitalized patients in Wuhan, China. Clin Infect Dis 71: 2089-2098, 2020.

20. Su M, Yuan J, Peng J, Wu M, Yang Y and Peng YG: Clinical prediction model for mortality of adult diabetes inpatients with COVID-19 in Wuhan, China: A retrospective pilot study. J Clin Anesth 66: 109927, 2020.

21. Wang F, Hou H, Wang T, Luo Y, Tang G, Wu S, Zhou H and Sun Z: Establishing a model for predicting the outcome of COVID-19 based on combination of laboratory tests. Travel Med Infect Dis 36: 101782, 2020.

22. Tian S, Liu H, Liao M, Wu Y, Yang C, Cai Y, Peng Z and Xiao SY: Analysis of mortality in patients with COVID-19: Clinical and laboratory parameters. Open Forum Infect Dis 7: ofaa152, 2020

23. Liu J, Li S, Liu J, Liang B, Wang X, Wang H, Li W, Tong Q, Yi J, Zhao L, et al: Longitudinal characteristics of lymphocyte responses and cytokine profiles in the peripheral blood of SARS-CoV-2 infected patients. EBioMedicine 55: 102763, 2020.
24. Wang L, Liu Y, Zhang T, Jiang Y, Yang S, Xu Y, Song R, Song M, Wang L, Zhang W, et al: Differentiating between 2019 novel coronavirus pneumonia and influenza using a nonspecific laboratory marker-based dynamic nomogram. Open Forum Infect Dis 7: ofaa169, 2020.

25. Soraya GV and Ulhaq ZS: Crucial laboratory parameters in COVID-19 diagnosis and prognosis: An updated meta-analysis. Med Clin (Barc) 155: 143-151, 2020

26. Thomas T, Stefanoni D, Dzieciatkowska M, Issaian A, Nemkov T, Hill RC, Francis RO, Hudson KE, Buehler PW, Zimring JC, et al: Evidence of structural protein damage and membrane lipid remodeling in red blood cells from COVID-19 patients. J Proteome Res 19: 4455-4469, 2020.

27. Wang K, Zuo P, Liu Y, Zhang M,Zhao X, Xie S, Zhang H, Chen X and Liu C: Clinical and laboratory predictors of in-hospital mortality in patients with coronavirus disease-2019: A cohort study in Wuhan, China. Clin Infect Dis 71: 2079-2088, 2020.

28. Sager R, Kutz A, Mueller B and Schuetz P: Procalcitonin-guided diagnosis and antibiotic stewardship revisited. BMC Med 15: 15, 2017.

29. Hu R, Han C, Pei S, Yin M and Chen X: Procalcitonin levels in COVID-19 patients. Int J Antimicrob Agents 56: 106051, 2020.

30. Lippi G and Plebani M: Procalcitonin in patients with severe coronavirus disease 2019 (COVID-19): A meta-analysis. Clin Chim Acta 505: 190-191, 2020.

31. Liu F, Li L, Xu M, Wu J, Luo D, Zhu Y, Li B, Song X and Zhou X: Prognostic value of interleukin-6, C-reactive protein, and procalcitonin in patients with COVID-19. J Clin Virol 127: 104370, 2020.

32. Fang FC, Naccache SN and Greninger AL: The laboratory diagnosis of COVID-19-frequently-asked questions. Clin Infect Dis, Jun 8, 2020 (Epub ahead of print).

33. Chen R, Sang L, Jiang M, Yang Z, Jia N, Fu W, Xie J, Guan W, Liang W, Ni Z, et al: Longitudinal hematologic and immunologic variations associated with the progression of COVID-19 patients in China. J Allergy Clin Immunol 146: 89-100, 2020.

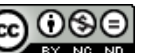

This work is licensed under a Creative Commons Attribution-NonCommercial-NoDerivatives 4.0 International (CC BY-NC-ND 4.0) License. 